\title{
The physician and AIDS education in the schools
}

WILLIAM M. KANE, PHD

\section{Physicians can be an excellent} resource for health education delivered in public and private schools. They are increasingly asked to participate in the development and delivery of AIDS education and prevention programs in schools. This paper provides the physician with information regarding health curriculum and resources which commonly exist in schools and makes suggestions for integrating AIDS education into the existing curriculum. Outcome objectives, teaching and learning strategies, and material for implementing a five-day, school-based AIDS education program are identified. Guidelines and recommendations to assist in preparing for the role of "guest speaker" in the schools are suggested.

Physicians increasingly will be asked by their communities to assist with AIDS education in local schools. Anticipating these requests, the American Osteopathic Association is continually providing scientific, clinical, and informational updates through JAOA and THE DO to ensure that knowledge concerning AIDS is current. In addition, the AOA provides physicians with audio and radio tapes to help improve their public speaking skills. To be an effective resource for AIDS education, physicians must understand the placement of such education within the overall school curriculum, as well as the expectations that schools may have of them.

The purposes of this article are to provide information to physicians regarding AIDS education in schools and to make suggestions on how physicians can interface with existing school efforts.

\section{Existing health education programs}

Guiding the development of the recommendations in this paper are the following assumptions:

Research conducted by the US Public Health Service $^{1}$ involving school-based health education programs shows that health education delivered within the context of a comprehensive approach is more effective than health education delivered in special assemblies, lec- tures, other curricula, or other ad hoc educational efforts.

Junior and senior high schools employ teachers with professional preparation in school health education. These teachers know content and educational strategies and have a general understanding of the role of health promotion and disease prevention in public health.

Each local school district has the autonomy to shape its curriculum content and to focus within parameters set forth by the state and federal government.

Students learn best when they are exposed to material via a variety of learning experiences. The physician lecture and follow-up question and answer period is only one learning experience among many.

Repetition and application of information is desirable and facilitates effective learning by students.

Students will have access to one semester of health education during both junior high and high school. These classes, which are taught by trained health educators, meet daily for 40-55 minutes. They focus on knowledge and skills that will help students to prevent disease and to develop healthy life-styles. They frequently cover the following general topics: nutrition education; prevention of unintended injuries and trauma; prevention of chronic diseases; prevention of infectious diseases; environmental, community, and public health; substance abuse prevention; utilization of health provider systems; development of positive mental health; physical fitness and health; and family life education.

\section{Positioning of AIDS education} within the school curriculum

The following recommendations are based on my current experience as Executive Director of the American College of Preventive Medicine, with a special interest in the physician's role in preventing disease, and on my past experience as a public school teacher with training in school health education.

Prevention of AIDS should be an important component of the content of the educational section on sexually transmitted diseases (STDs), which would 
At the completion of the AIDS prevention component, students will be able to do the following:

(1) Explain the history and current epidemiology of AIDS

(2) Identify which activities and behavior put an individual at high risk of acquiring the HIV virus.

(3) Identify actions that individuals can take to prevent AIDS.

(4) Debunk common myths regarding AIDS, and evaluate the accuracy of sources of AIDS information.

(5) Identify public policy issues raised by the AIDS epidemic, and, citing current science literature to support points made, discuss in depth at least one of the issues.

(6) Identify sources of accurate AIDS information, professional counseling, and medical advice.

TABLE 2. TEACHING AND LEARNING STRATEGIES FOR AIDS EDUCATION.

The prevention of AIDS component would involve the following mix of teaching and learning experience during the five class periods:
Pre-test

Question-and-answer periods

Small-group work sessions

Reading assignment

Out-of-class project

Guest speaker (physician)
Group discussion

Lecture

Physician worksheet

Movie

Post-test be part of the unit on prevention of infectious diseases. This unit should be covered in 14 days (one 40-55 minute class per day). Because of the high incidence, the STD/AIDS component should occupy eight of those 14 days, with prevention of AIDS content filling five of the eight class periods. Homework assignments should accompany the classroom activities.

The recommendation that AIDS education should be included in the prevention of infectious diseases unit rather than be a major component of the family life education unit may be contrary to recommendations of others. STDs and AIDS are epidemic infectious diseases for which teenagers are or will be at high risk, and, therefore, primary learning about these diseases should be in the prevention of infectious diseases unit. Family life education is an important part of a comprehensive health education experience. This unit focuses on the importance of the family, loving relationships among family members, responsibilities of family members, human sexuality, pregnancy, childbirth and raising families, and family planning. AIDS and other STDs, which would be mentioned as a possible consequence of indiscriminate sexual behavior, do not play a major role in the healthy family life context. Likewise, AIDS also would be mentioned as a possible consequence of intravenous
TABLE 3, DAILY CLASSROOM ACTIVITIES MODEL FOR FIVE-DAY AIDS EDUCATION COMPONENT.

Day 1 Pre-test

Assign each student to write and submit anonymously three topics about AIDS that the class should cover

Present and discuss student learning objectives.

Assign and discuss out-of-class project.

Assign reading.

Day 2 Modify objectives based on student questions. Present 20-minute lecture.

Have class discussion and question-and-answer session.

Give small-group worksheet assignment (problem solving).

Day 3 Review objectives of the film on AIDS.* Show "The AIDS movie."

Discuss and review major points of movie.

Day 4 Present guest speaker on AIDS (physician).

Day 5 Review speaker's points from the previous day. Review student objectives.

Post-test.

Speak individually with students during test regarding outside projects, which will be submitted at a later date.

*The recommended movie is "New Day," which can be obtained from Durrin Films, 22 Riverside Dr, Wayne, NJ 07470-3191.

drug use in the unit on substance abuse prevention, and it would also be part of the environmental, community, and public health unit.

The health education program should be scheduled to cover prevention of infectious diseases prior to the family life education, substance abuse prevention, and environmental, community, and public health units. These units would cross-refer to the prevention of infectious disease unit if further questions arise.

\section{Goals and teaching and learning methods}

The objectives of AIDS education in schools are outlined in Table 1. Teaching and learning strategies are listed in Table 2, and a model for daily class activities for the five-day AIDS component is presented in Table 3.

\section{The physician's role}

The physician lecture and following question-andanswer period are important parts of the AIDS education unit. When a physician is invited into the classroom, the teacher should provide suggestions that will ensure that the presentation will complement, reinforce, and add to the learning that has already taken place. It is necessary to know what learning experience preceded the physician's presentation and what follow-up sessions will include. 
If the teacher does not volunteer that information, the physician should ask. Physicians are encouraged to use the CDC guidelines ${ }^{2}$ for AIDS school health education when preparing for the teaching session. Once the physician has an understanding of the situation, the real challenge begins. The following are the physician's responsibilities:

\section{Identify what information the students are to} learn as a result of the lecture and question and answer session. Do not attempt to cover a large amount of information. If the students learn three or four important points, the visit will have been a success. Write those points down. Review them with the students prior to the lecture and leave them with the teacher.

Lecture for approximately 20 minutes. If you are a good speaker and the topic is interesting (which it is), you will be able to maintain most students' attention for that period of time. You will find that the question-and-answer session is more productive than the lecture.

Summarize the main points at the end of the lecture.

Open the class to questions. Be prepared to ask your own questions - "A question I commonly get is...."-in order to start the discussion moving. You may wish to prime the teacher with a question or two. Repeat any question you are asked and probe to make sure that you understand exactly what the student is asking. Sometimes, because the student is misinformed, the question will not make sense. By eliciting further information, you will able to better understand what the student wants to know.

Summarize the major points again at the end of the class. The benefits of repetition cannot be overemphasized. Leave a copy of the student learning objectives with the teacher. In addition, you may wish to leave with the teacher a one-page worksheet that questions students about the main points of the presentation. This will provide one more opportunity to reinforce the message.

\section{Summary}

The American Osteopathic Association encourages physicians to assume the role of community spokesperson on AIDS. Education and training provided by the AOA prepares the physician for public speaking and gives a current update on the AIDS epidemic.

This paper provides background information regarding AIDS education in schools and assists the physician in preparing to serve as an expert resource for students. It must be emphasized that each local community will approach the AIDS issue differently. However, because prevention of AIDS is such a critical necessity, it is the physician, as one who has expert knowledge and is viewed as an opinion leader in the community, whose responsibility it is to urge schools to approach the prevention of AIDS in a responsible manner.

1. Connell DB, Turner RR, Mason EF: Summary of findings of the School Health Education: Health promotion effectiveness, implementations, and costs. J School Health 1985;155:316-321.

2. Guidelines for effective school health education to prevent the spread of AIDS. MMWR 1988;37:(suppl)1-13.

From the American College of Preventive Medicine, where Dr Kane is Executive Director.

Reprint requests to Dr Kane, American College of Preventive Medicine, 1015 15th St NW, Suite 403, Washington, DC 20005. 


\section{When the Goal Is to Reduce Elevated Blood Cholesterol. . . .}
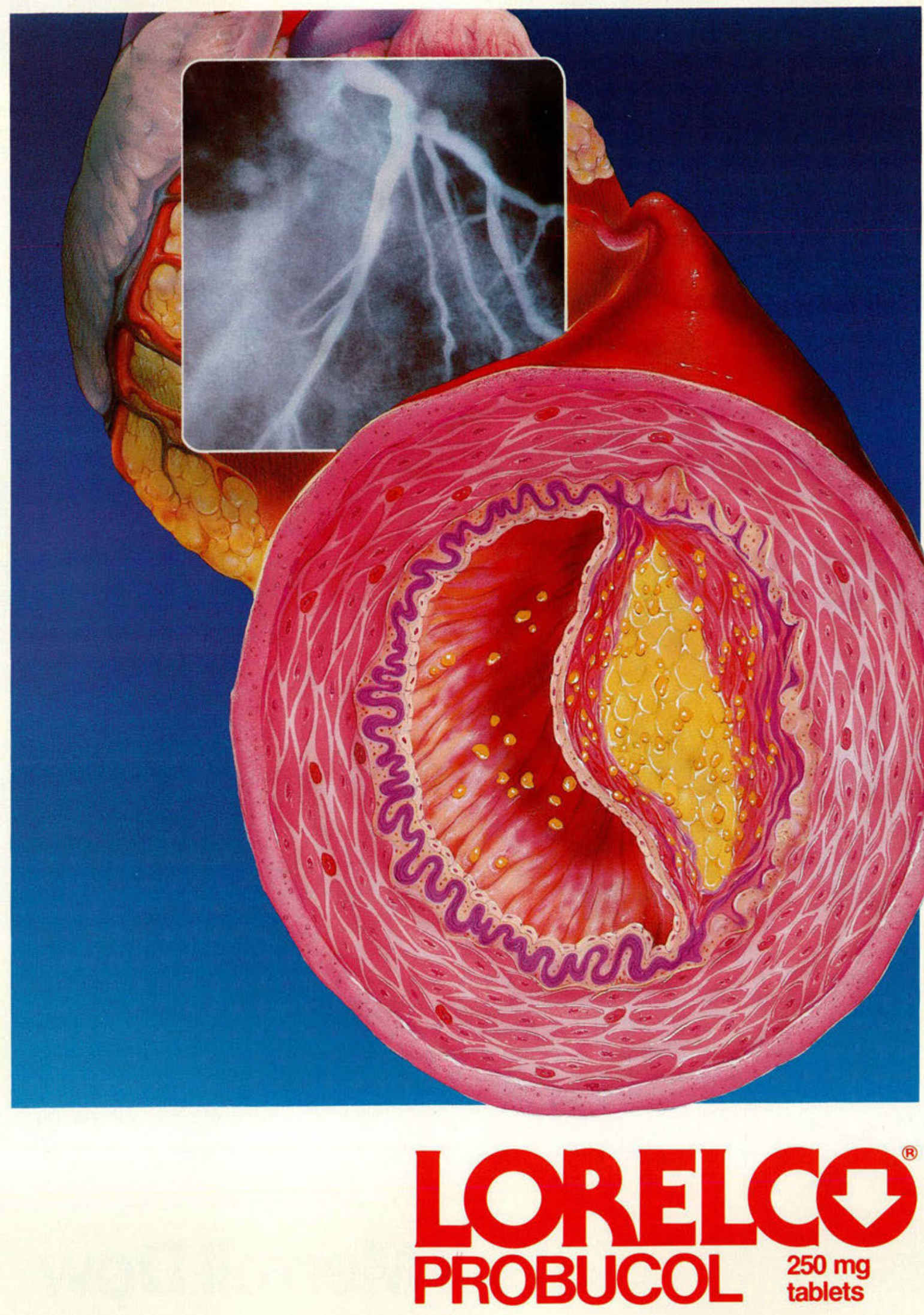

Prescribing Information appears on the last page. 


\section{Choose}
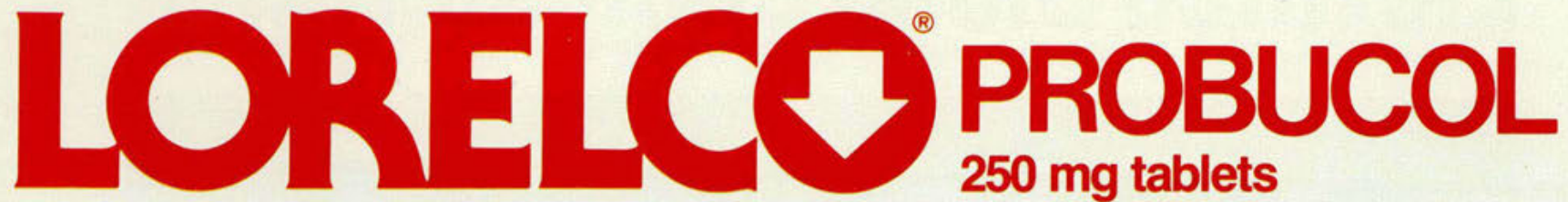

$250 \mathrm{mg}$ tablets

as an adjunct to diet

\section{Effectively}

Lowers Serum

Cholesterol

with a Unique

Combination

of Benefits

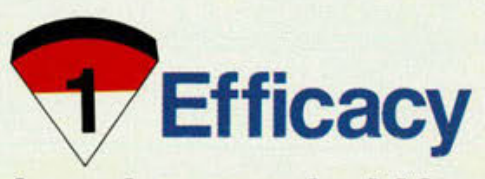

Lorelco works! You can prescribe it with confidence. Lorelco can virtually double the cholesterol-lowering efficacy of diet alone. ${ }^{1}$ Total cholesterol-lowering efficacy beyond diet ranges from $10.7 \%$ to $27 \%$ in reported studies. ${ }^{1-4}$

\section{(2) Indications}

Lorelco is specifically indicated for the reduction of elevated serum cholesterol in patients with primary hypercholesterolemia who have not responded adequately to diet.

Consider the Whole Picture.

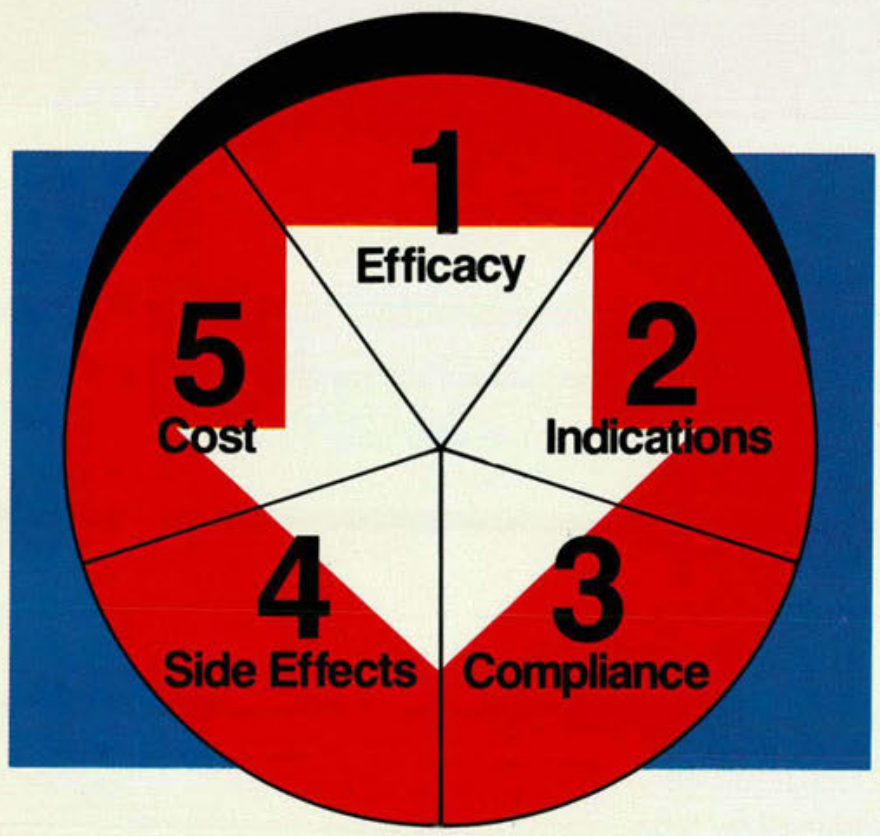




\section{References:}

1. LeLorier $\mathrm{J}$, et al: Diet and probucol in lowering cholesterol concentrations: additive effects on plasma cholesterol concentrations in patients with familial type II hyperlipoproteinemia. Arch Intern

2. Mellies MJ, et al: Effects of probucol on plasma cholesterol, high and low density lipoprotein cholesterol, and apolipoproteins A1 and A2 in adults with primary familial hypercholesterolemia.
Metabolism 1980; $29: 956-964$.

3. Polachek AA, et al: Probucol in the long-term treatment of hypercholesterolemia. Curr Med Res Opin 1973: 1:323-330

\section{Lorelco ${ }^{\circledR}$ Tablets}

\section{(probucol tablets)}

CAUTION: Federal law prohibits dispensing without prescription.

DESCRIPTION: Lorelco (probucol) film-coated tablets for oral administration contain $250 \mathrm{mg}$ of probucol per tablet. Each tabiet also contains as inactive ingredients: corn starch, ethylcellulose, olycerin, hydroxypropyl cellulose, hydroxypropy
methylcellulose 2910 , iron oxide, lactose, magnesium stearate, microcrystalline cellulose, polysorbate 80 , talc, and titanium dioxide. Lorelco is an agent for the reouction of elevated serum cholesterol. The chemical name is $4.4^{\circ}$-(isopropylidenedithio bis $(2,6-d i-1-b u t y / p h e n o l)$. Its chemical structure does not resemble that of any other available cholesterol-lowering agent. It is

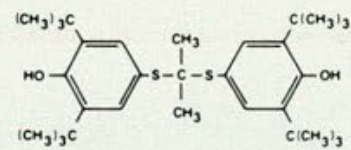

CLINICAL PHARMACOLOGY: Lorelico (probucol) lowers serum cholesterol and has relatively little effect on serum triglycerides. Patients responding to probucol exhibit a decrease in low density lipoprotein cholesterol. Cholesterol is reduced not only in the low density lipoproten fraction, but also in some high density lipoprotein fractions with proportionately greater
effect on the high density portion in some patients. Epidemiological studies have shown that both low HDL-cholesterol and high effect on the high density portion in some patients. Epidemiological studies have shown that both low HDL-cholesterol and high
LOL-cholesterol are independent risk tactors for coronary heart disease. The fisk of lowering HOL-cholesterol while lowering
LLL-cholesterol remains unknown. There is little or no effect reported on very low density lipoprotein.

Studies on the mode of action of Lorelco indicate that it increases the fractional rate of catabolism of low density lipoproteins This effect may be linked to the observed increased excretion of fecal bile aciods. a final metabolic pathway for the elimination absorption of dietary cholesterol. There is no increase in the cyclic precursors of cholesterol namely desmosterol and 7 .

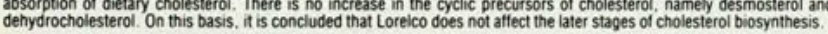
Absorption of Lorelco from the gastrointestinal tract is limited and variable. When it is administered with food, peak blood levels are higher and less variable. With continuous administrabon in a dosage of $500 \mathrm{mg}$ b. . . . The blood levels of an individua
gradually increase over the first three to four months and thereatter remain tairly constant. in 116 patients treated with Lorelco for periods of three months to one year, the mean blood level was $23.6 \pm 17.2 \mathrm{mcg} / \mathrm{ml}( \pm S .0$.$) ranging to 78.3 \mathrm{mcg} / \mathrm{ml}$ l. Levels
observed after seven years of treatment in 40 patients yieided an average value of $21.5 \pm 16.5 \mathrm{mcg} / \mathrm{ml}( \pm S .0$.) ranging to 62.0

At the end of 12 months of treatment in eight patients. blood levels averaged $19.0 \mathrm{mcg} / \mathrm{ml}$. Six weeks after cessation of therapy. the average had talien

In December 1984 a National Institutes of Health Consensus Development Conference Paneli concluded that lowering definitely
elevated blood cholesterol levels (specifically blood leveis of low-density lipoprotein cholesterol) will reduce the risk of heart alevated biood cholesterol levels (specifically blood levels of low-density lipoprotein cholesterol) Will reduce the risk of hear years may be required betore ongoing long-term investigations will resolve this question.

INDICATIONS AND USAGE: Serious animal toxicity has been encountered with probucol. See WARNINGS and ANIMAL INDICATIONS and WARNINGS

Drug therapy should not be used for the routine treatment of elevated blood lipids for the prevention of coronary heart disease
Dietary therapy specific for the type of hyperlipidemia is the initial treatment of choice. Excess body weight may be an important actor and should be addreseed prior to any dide disease such as hypothyroidism or diabetes melilutus should be looked for and adequately treated. The use of drugs should be considered only when reasonabie attempts have been made to obtain satistactory results with non-drug methods. II the decisio
ultimately is to use drugs, the patient should be instructed that this does not reduce the importance of adhering to det The selection of patients for cholesterol-lowering drug therapy should take into account other important coronary risk factors such as smoking. hypertension and diabetes melitus. Consideration should be given to the efficacy. saletry and compliance Lorelco may be indicated for the reduction of elevated serum cholesterol in patients with primary hypercholesterolemia (elevated low density lipoproteins) who have not responded adequately to diet. weight reduction and control of diabetes mellitus. Lorelco may be useful to lower elevated cholesterol that occurs in patients with combined hypercholesterolemia and hyper It is not always possible to predict from the lipoprotein type or other factors which patients will exhibit tavorable results. Lipid levels should be periodically assessed. Small or transient changes in high density lipoprotein cholesterol may be due to The effect of probucol-induced reduction of serum cholesterol or triglyceride levels or alteration of HOL-cholesterol levels on morbidity or mortality due to coronary heart disease has not yet been established. Several years may be required belore ongoing CONTRAINDICATIONS: (See also PRECAUTIONS) Loreico is contraindicated in patients who are known to have a WARNINGS: SERIOUS ANIMAL TOXICITY HAS BEEN ENCOUNTERED WITH PROBUCOL IN RHESUS MONKEYS FED AN

Although Q-T prolongation can occur in patients on probucol, the arrthythmias observed in monkeys fed large doses of probucol
added to an atherogenic diet have not been reported in man; nevertheless, the following precautions are deemed prudent. 1. At the start of treatment with Lorelco and throughout the treatment period, patients should be advised to adhere to a low cholesterol, low tat diet

2. As part of an overall evaluation, a baseline, six month, and one year repeat ECG tracing should be considered. If marked
proiongation of the Q $-\mathrm{T}$ interval (after correction for rate) occurs, the possible benefits and risks should be carefully considered betore making the decision to continue the probucol administration

Lorelco should not be used in patients with evidence of recent or progressive myocardial damage or findings suggestive of ventricular arrhythmias.

No instances of increase in ectopy attributed to Lorelco have been reported. However, patients with unexplained syncope while PRECAUTIONS General: Because Lorelco is intended for long-term administration, adequate baseline studies should be pertormed to
determine that the patient has elevated serum cholesterol levels Serum lipid levels should be determined before treatment and
repeated during the first few months of treatment and periodically thereafter. A tavorable trend in cholesterol reduction should be repeated during the first few months of treatment and periodically thereafter. A tavorable trend in cholesterol reduction should be
evident during the first three to four months of administration of Lorelco. Probucol lowers serum total and L L cholesterol, and also lowers $\mathrm{HLL}$-cholesterol in many patients with elevated $L \mathrm{DL}$-cholesterol. Epidemiologic studies within hypercholesterolemic populations have shown that serum HOL-cholesterol is an independent, inversely correlated, risk factor for coronary hear
disease isee CLINICAL PHARMACOLOGY) Animal or human studies which could confirm or deny the hypothesis that drug induced alteration in HOL-cholesterol aftects cardiovascular nisk have not been carried out If is not known whether probuco.
induced reduction of serum HDL-cholesterol will affect cardiovascular risk since no long-term, controlled clinical trials of
probucol for the prevention of coronary heart disease, similar to the LRC-CPPT (See CLINICAL STUDIES). have been pertormed probucol for the prevention of coronary heart disease, similar to the LRC-CPPT (See CLLNICAL STUDIES), have been performed The probable benefits obtained from LDL-cholesterol reduction must be weighed against the possible risk of a reduction in HDL-
cholesterol when assessing the response of each patient receiving Loreico treatment. If satistactory lipid alteration is not Information for Patients: The patient should be instructed to adhere to a prudent diet. Females should be cautioned against
becoming pregnant for at least six months after discontinuing Lorelco and should not breast feed their infants during therapy
with Lorelico.

Laboratory Tests: The physician should schedule periodic blood lipid determinations and should consider periodic Elevations of the serum transaminases (glutamic-oxalacetic and glutamic-pyruvic), bilirubin, alkaline phosphatase, creatine phosphokinase. uric acid, blood urea nitrogen and blood glucose above the normal range were observed on one or more patient's clinical state or other modes of therapy. Although the basis for the relationship between probucol and these abnormalities is not firm, the possibility that some of these are drug-related cannot be excluded In the controfled trials, the incidence of abnormal laboratory values was no higher in the patients treated with probucoli than in the patients who recerved
placebo. If abnormal laboratory tests persist or worsen, if clinical signs consistent with the abnormal laboratory tests develop.

Drug Interactions: The addition of clofibrate to probucol is not recommended since the lowering effect on mean serum levels of either $\mathrm{LDL}$ or total cholesterol is generally not significantly additive and, in some patients, there may be a pronounced
lowering of HDL-cholesterol. Nerther oral hypoglycemic agents nor oral anticoagulants alter the effect of Lorelco on serum lowering of HDL-cholesterol. Neither oral hypoglycenic agents nor oral anticoagulants alter the effect of Lorelco on servum
cholestero. The dosage of these agents is not usually modified when given with Lorelco. Monkeys fed a high fat. . high

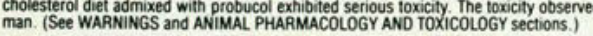

Baker SG, et al: Treatment of homozygous familial hypercholesterolaemia with probucol. S Afr Med J 1982; $62: 7-11$

5. McCaughan D: The long-term effects of probucol on serum lipid levels. Arch Intern Med 1981; $1428-1432$

6. Data on file. MERRELL DOW PHARMACEUTICALS INC., Cincinnati, Ohio 45242
Carcinogenesis, Mutagenesis, Impairment of Fertility

In chronic studies of two years duration in rats, no toxicity or carcinogenicity was observed. These results are consistent with Pregnancy

Pregnancy - Category 8: Reproduction studies have been pertormed in rats and rabbits at doses up to 50 times the human and well-controlled studies inence of impaired fertility or harm to the fetus due to probucol. There are, however, no adequate

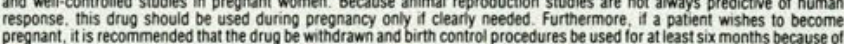
persistence of the drug in the body for prolonged periods. (See CLINICAL PHARMACOLOCY.)

Labor and Delivery: The effect of Lorelco on human labor and delivery is unknown.

Nursing Mothers: It is not known whether this drug is secreted in human milik, but it is likely to be since such excretion has

Pediatric Use: Satety and effectiveness in children have not been established

ADVERSE REACTIONS

Gastrointestinal

Calcea or loose stools, flatulence, abdominal pain, nausea. vomiting, indigestion, gastrointestinal bleeding

Cardiovascular

ion of the Q-T interval on ECG

Neurologic

Hematologic

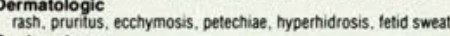

Genitourinary

Ophthalmic

coniunctivitis, tearing, blurred vision

Endocrine
enlargement of multinodular goiter

Idiosyncrasies

An idiosyncratic reaction observed with initiation of therapy and characterized by dizziness, palpitations, syncope, nausea vomiting and chest pain has been observed.

Other diminished sense of taste and smell, anorexia, angioneurotic edema

DRUG ABUSE AND DEPENDENCE: No evidence of abuse potential has been associated with Lorelco, nor is there .

OVERDOSAGE: There is a single report of a $15 \mathrm{~kg}$, three-year-old male child who ingested $5 \mathrm{gm}$ of probucol. Emesis was induced by ipecac. The child remained well, apart from a brief episode of loose stools and flatulence. No specific information is available on the treatment of overdosage with Lorelco and no specific antidote is available. Probucol is not dialyzable.
Treatment is symotomatic and supportive. Probucol has shown no identifiable acute toxicity in mice and rats. In these animals the LO $\mathrm{Ls}_{\mathrm{s}}$ (oral) is in excess of $5 \mathrm{gm} / \mathrm{kg}$ of body weight.

DOSAGE AND ADMINISTRATION: For adult use only. The recommended and maximal dose is $500 \mathrm{mg}$ (two tablets of $250 \mathrm{mg}$ each) twice daily with the morning and evening meals.

HOW SUPPLIED: Each white film-coated tablet contains $250 \mathrm{mg}$ probucol and is imprinted with the DOW diamond trademark over the code number 51, or LORELCO 250 .

Keep well closed. Store in a dry place. Avoid excessive heat. Dispense in well closed light-resistant container with childesistant closure.

ANIMAL PHARMACOLOGY AND TOXICOLOGY: In rhesus monkeys administration of probucol in diets containing Premonitory syncope was frequently observed and was associated with a pronounced prolongation of the Q-T intervals ( 30 to 50 percent longer than that observed in untreated monkeys). Serum levels of probucol greater than $20 \mathrm{mcg}$ mi were generally interval from control values was usually seen at $40 \mathrm{mcg} / \mathrm{ml}$ and above. Blood levels in humans receiving probucol average

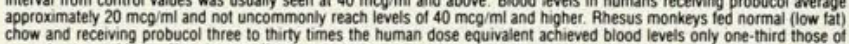
many human subjects. No adverse effects were detected in these monkeys over an eight-year period of continuous drug administration. In another study in rhesus monkeys, an atherogenic diet was ted for two years and daily treatment with probucol. separated in time from the atherogenic meal, was carried out during the second year. Serum probucol levels
ranged 20 to $50 \mathrm{mc} / \mathrm{ml}$ in five of ten monkeys, and less in the remaining animals. Marked prolongation of the $Q-\mathrm{T}_{\mathrm{C}}$ interval in the electrocardiogram or syncopal behavior was never observed over the entire one-year treatment period. Regression of animals receiving probucol. It should be emphasized that both $\mathrm{HOL}$-cholesterol and LOL-cholesterol were markedly reduced in this regression study. During the performance of a two-year chronic study involving 32 probucol-treated dogs (beagles)
there were 12 tatalities. Subsequent experiments have indicated that probucol sensitizes the canine myocardium to eppnephrine, resulting in ventricular fibrilitition in many dogs. Among the animal species in which probucol has been studied. the dog is peculiar with respect to the phenomenon of sudden death due to the sensitization of the myocardium to
epinephrine. In contrast to findings in the dog. injections of epinephrine to probucol-treated monkeys did not induce ventricular fibrillation.

In other studies, monkeys were given probucol either before and after, or only after myocardial intarction induced by
coronany artery ligation. In these studies there was no difterence between probucol-and placebo-treated groups with respect to either survival or detailed blind quantitation of myocardial changes (gross and histopathologic).

Probucol has shown no identifiable toxicity in mice and rats. In these animals the $L D_{50}$ (oral) is in excess of five $9 \mathrm{~m} / \mathrm{kg}$ of body weight. In chronic studies of two-year duration in rats, no toxicity or carcinogenicily was observed.

From studies in rats, dogs and monkeys, it is known that probucol accumulates slowly in adipose fissue. Approximately 90
percent of probucol administered orally is unabsorbed. For that which is absorbed, the biliary tract is the major pathway for clearance from the body and very little is excreted by way of the kidneys.

Myocardial injury was produced in various groups of rats by one of the following procedures: aortic coarctation, coronary
ligation, or cobalt of isoproterenol injection. After probucol adrininistration, no deleterious effects related to treatment occurred as measured by survival and microscopic examination of myocardial damage.

Probucol was administered to minipigs beginning ten days betore ligation of coronary artery and continued for 60 days post
surgery. Challenge with epinephrine at the end of 60 days tailed to induce ventricular firrillation in any of the coronary. ligated. probucol-treated minipigs

CLINICAL STUDIES: In a multicenter, randomized, double-blind study, the LRC-CPPT2, hypercholesterolemic patients treated with an oral bile acid sequestrant (cholestyramine) and a cholesteroi lowering diet experienced average total and lowdensity lipoprotein cholesterol reductions greater than those obtained in the placebo group treated with diet alone. The
cumulative seven-year incidence of the primary end point--combined incidence of definite $\mathrm{CHD}$ death and or definite nonfatal cumulative seven-year incidence of the primarry end point-combined incidence of definite CHD death and or definite nonfatal
myocardial intarction-was $7 \%$ in the cholestyramine group and $8.6 \%$ in the placebo group. This was a $19 \%$ reduction in
fisk (P less than 0.05 , single-tail test) of the primary end point reflecting a $24 \%$ reduction in definite CHD death and a $19 \%$ reduction in nontatal myocardial infarction.

The subjects included in the study were middle-aged men ( $35-59$ years old) with serum cholesterol levels at least 265 mg/d: and no previous history of heart disease. It is not

The bile acid sequestrant, cholestyramine was used in the above trial. Caution should be exercised in extrapolating these results to probucol since it differs trom cholestyramine with regard to its mode of action. spectrum of cholesterol-lowering potency, effect on $\mathrm{HDL}-$ cholesterol and possible toxicity. The effect of probucol-induced reduction of serum cholesterol levels REFERENCES:

. Consensus Development Panel, Lowering blood cholesterol to prevent heart disease. JAMA 253: 2080-2086, 1985

The Lipid Research Clinics Program. The Lipid Research Clinics coronary primary prevention trial results. I. Reduction in

Product information as of April, 1986

Y196D

MERRELL DOW PHARMACEUTICALS INC.
Subsidiary of The DOw Chemical COmpany

\section{Merrell Dow}

